\title{
Risk of Thromboembolic Events after Perioperative Chemotherapy Versus Surgery Alone for Esophageal Adenocarcinoma
}

\author{
Roy J. J. Verhage, MD ${ }^{1}$, Sylvia van der Horst, MSc $^{1}$, Pieter C. van der Sluis, MD ${ }^{1}$, \\ Martijn P. J. K. Lolkema, MD, $\mathbf{P h D}^{2}$, and Richard van Hillegersberg, $\mathbf{M D}$, $\mathbf{P h D}^{1}$ \\ ${ }^{1}$ Department of Surgery, University Medical Center Utrecht, Utrecht, The Netherlands; ${ }^{2}$ Department of Medical Oncology, \\ University Medical Center Utrecht, Utrecht, The Netherlands
}

\begin{abstract}
Background. Major oncologic surgery is associated with a high incidence of thromboembolic events (TEE). Addition of perioperative chemotherapy in esophageal cancer surgery may increase the risk of TEE.

Methods. The thromboembolic toxicity profile was analyzed in patients with esophageal adenocarcinoma (EAC). Two groups were identified: patients who underwent esophagectomy and received perioperative chemotherapy with epirubicin, cisplatin, and capecitabine (ECC; $n=52$ ), and patients who were treated with surgery alone $(n=35)$. Results. A total of 22 TEEs was observed in 17 patients $(32.7 \%)$ in the chemotherapy group and 3 patients $(7.5 \%)$ in the surgery-alone group $(P<.01)$. The relative risk of developing a TEE for patients receiving perioperative chemotherapy during the whole treatment period was 3.8 (95\% confidence interval 1.2-12.0). A preoperatively occurring TEE did not increase the risk of postoperative TEE, nor did it increase postoperative hospital stay $(P=.325)$. Median postoperative hospital stay was 23 days (range 14-78) for patients with a postoperative TEE and 15 days (range 10-105) for patients without TEE $(P=.126)$. Perioperative chemotherapy with the epirubicin, cisplatin, and capecitabine regimen was independently associated with the development of TEE in the combined preoperative and postoperative period $(P=.034)$.

Conclusions. Perioperative chemotherapy improves survival for operable esophageal cancer but comes at the price
\end{abstract}

(C) The Author(s) 2011. This article is published with open access

at Springerlink.com

First Received: 18 April 2011;

Published Online: 12 August 2011

R. J. J. Verhage, MD

e-mail: R.J.J.Verhage@umcutrecht.nl of toxicity. Perioperative chemotherapy for EAC increases the risk of TEE. However, chemotherapy-related preoperative TEE did not increase the risk of postoperative TEE, nor did it increase postoperative hospital stay, justifying its use in clinical practice.

In the Western world, esophageal adenocarcinoma (EAC) has become the most prevalent type of cancer in the esophagus, overtaking squamous cell carcinoma. ${ }^{1-3}$ Perioperative treatment of patients with operable adenocarcinoma of the distal esophagus, gastroesophageal junction, and stomach with chemotherapy consisting of epirubicin, cisplatin, and 5-fluorouracil improves overall survival in patients when compared to surgery alone. ${ }^{4}$ The regimen consists of three preoperative treatment cycles followed by surgery and three postoperative cycles. Oral administration of capecitabine as an alternative for prolonged intravenous infusion of 5-fluorouracil showed equal efficacy in patients with advanced or inoperable esophagogastric cancer. ${ }^{5}$ The epirubicin, cisplatin, and capecitabine (ECC) regimen may be used as a substitute for the epirubicin, cisplatin, and 5-fluorouracil regimen. ${ }^{6}$ Our current standard for patients with operable esophageal and gastroesophageal junction adenocarcinoma is perioperative chemotherapy with the ECC regimen combined with radical esophagectomy and gastric conduit reconstruction.

Gastroesophageal cancers are associated with the highest risk of venous thrombosis. ${ }^{7}$ The reported incidence of thromboembolic events (TEE) during chemotherapy for advanced gastroesophageal cancer is approximately $12 \%$ and even higher when patients are treated with cisplatin. ${ }^{8}$ Another important risk factor for TEE is major (oncologic) surgery. ${ }^{9,10}$ 
Still, reports of TEE during chemotherapy are limited to series of patients with advanced inoperable gastroesophageal cancer. Little is known about the incidence and consequences of TEE during perioperative chemotherapy in patients with cancer of the upper gastrointestinal tract. This study shows our experience of the past 4 years during which the ECC protocol was applied in patients with EAC. The thromboembolic toxicity profile of patients receiving perioperative ECC chemotherapy was analyzed and compared with a group of patients from the same time period who were treated with surgery alone.

\section{METHODS}

\section{Data Collection}

A prospective database of all esophageal resections in our tertiary referral center (University Medical Center Utrecht, Utrecht, the Netherlands) is collected continuously. The research protocol was in accordance with the guidelines of the medical ethics committee. Database entries include standard patient characteristics, as well as prospectively collected intra- and postoperative data. The database enables entry of various complications, among which deep venous thrombosis, pulmonary embolism, and myocardial infarction form separate entries. Atrial fibrillation was also documented in the database because this condition is associated with both esophagectomy and an increased risk of postoperative thromboembolic complications. Complications that occurred during preoperative chemotherapy were retrieved from the patient records of the medical oncology department.

\section{Inclusion Criteria}

The study population consisted of all consecutive patients who underwent esophageal resection with gastric conduit reconstruction for EAC complemented with perioperative ECC therapy (January 1, 2007, to February 1, 2011). To eliminate historical bias, the control group comprised patients who underwent the same surgical treatment without chemotherapy in that same time period (surgery-alone group). Reasons for not commencing preoperative chemotherapy were $\mathrm{T} 1$ disease, weight loss $>10 \%$, World Health Organization performance status $>2$, and Groningen Frailty Index $>3$ (for patients above 70 years of age). ${ }^{11,12}$

\section{Chemotherapy}

Patients eligible for perioperative ECC chemotherapy received three preoperative treatment cycles and another three postoperative treatment cycles. One chemotherapy cycle consisted of intravenous administration of epirubicin and cisplatin on day $1\left(50\right.$ and $60 \mathrm{mg} / \mathrm{m}^{2}$, respectively), followed by $625 \mathrm{mg} / \mathrm{m}^{2}$ of capecitabine twice daily for 21 days. Adaptations to the regimen (i.e., dose reduction or change of regimen) were applied when necessary on the basis of the occurrence of adverse events during therapy as defined by the Common Terminology Criteria for Adverse Events (CTCAE) and the Common Toxicity Criteria. ${ }^{13}$

After completion of the second cycle, a computed tomography (CT) scan was performed to evaluate response. In case of tumor progression, a third cycle was not administered, and surgery was rescheduled accordingly. In case of a TEE during preoperative chemotherapy, anticoagulative therapy was initiated with low-molecular-weight heparin (Fragmin, Pfizer, New York, NY; daily; subcutaneous; <80 kg, $2500 \mathrm{IU} ;>80$ kg, $5000 \mathrm{IU})$.

\section{Surgical Therapy}

Surgical therapy consisted of esophageal resection with gastric conduit reconstruction through a transthoracic or transhiatal approach performed by a single experienced surgeon. Preferably, esophagectomy was performed with the use of minimally invasive surgery. Transthoracic surgery included two-field lymphadenectomy. Abdominal lymphadenectomy was similar for all patients (truncal and perigastric). Operative approach was matched to patient physiology and tumor characteristics. All esophagogastric anastomoses were hand sewn and situated cervically on the left side. Perioperatively, all patients received standard thrombotic prophylaxis with low-molecular-weight heparin (Fragmin, Pfizer; daily; subcutaneous; $<80$ kg, 2500 IU; $>80 \mathrm{~kg}, 5000 \mathrm{IU})$ and stockings.

\section{Primary Outcome}

The primary outcome was defined as the occurrence of a TEE during one of three distinct periods, as follows: (1) the preoperative period, defined as the day of diagnosis until the day of surgery; (2) the postoperative period, defined as during the hospital stay or until the 30th day after surgery; and (3) the postoperative chemotherapy period, from the day on which postoperative chemotherapy was resumed until 30 days after the last administration of chemotherapy. The latter period was only applicable to those patients who received postoperative chemotherapy. TEEs were defined and graded according to CTCAE version 4.03. ${ }^{13}$ Only events graded 2 or higher were included for analysis (Table 1). 
TABLE 1 TEE grades as defined by the CTCAE

\begin{tabular}{ll}
\hline $\begin{array}{l}\text { CTCAE } \\
\text { grade }\end{array}$ & Definition \\
\hline $1^{\text {a }}$ & Venous thrombosis (e.g., superficial thrombosis) \\
2 & Venous thrombosis (e.g., uncomplicated deep vein thrombosis), medical intervention indicated \\
3 & $\begin{array}{c}\text { Thrombosis (e.g., uncomplicated pulmonary embolism [venous], nonembolic cardiac mural [arterial] thrombus), medical } \\
\text { intervention indicated }\end{array}$ \\
4 & $\begin{array}{c}\text { Life-threatening (e.g., pulmonary embolism, cerebrovascular event, arterial insufficiency), hemodynamic or neurologic instability, } \\
\text { urgent intervention indicated }\end{array}$ \\
& Death
\end{tabular}

${ }^{a}$ Grade 1 not included for analysis

\section{Statistical Analysis}

Data were analyzed by SPSS statistical software, version 15.0 (SPSS, Chicago, IL). Cross-tabulation with chi-square testing was used to identify differences between cohorts regarding baseline characteristics and the occurrence of TEE during separate and combined treatment periods. Student's $t$-test was used for continuous variables. Associations between risk factors and TEE were analyzed with univariate regression analysis. Multivariate logistic regression was applied to correct for confounders. The following risk factors and possible confounders with respect to the development of TEE were identified before analysis: age above 60 years, gender, body mass index above 27, American Society of Anesthesiologists score, chronic obstructive pulmonary disease (COPD), diabetes, hypertension, previous TEE, history of smoking, length of hospital stay, operative approach, operative time, presence of tumor-positive lymph nodes, and tumor differentiation grade. Anticoagulant use at the time of diagnosis was included as a protective factor. A $P$-value of $<.05$ was considered statistically significant.

\section{RESULTS}

Between January 1, 2007, and February 1, 2011, a total of 107 patients underwent esophagectomy with gastric conduit reconstruction for EAC. After exclusion of patients who received chemoradiotherapy or primary chemotherapy other than ECC, 87 patients remained. Mean age was 65 years, and the ratio of men to women was approximately $4: 1$.

\section{Cohorts and Baseline}

Perioperative chemotherapy in the form of ECC was administered to 52 patients. The remaining 35 patients did not receive chemotherapy. Reasons for not giving chemotherapy are presented in Table 2.
TABLE 2 Reasons for not receiving neoadjuvant chemotherapy in 35 of 87 patients

\begin{tabular}{lr}
\hline Characteristic & $n$ \\
\hline Patient unfit for perioperative chemotherapy $^{\mathrm{a}}$ & 17 \\
Previous chemotherapy with serious toxicity & 2 \\
Weight loss $>10 \%$ & 2 \\
Refused patient consent & 2 \\
Esophageal perforation requiring immediate intervention & 1 \\
Early-stage disease $(\mathrm{uT} \leq 1)$ & 11
\end{tabular}

${ }^{a}$ Patients with a World Health Organization performance status of $>2$ (corresponding to a Karnofsky score of $<70$ ) or a Groningen Frailty Index $>3$ (for patients older than 70)

Both age and gender were evenly distributed between the surgery-alone group and the group receiving ECC. In the surgery-alone group, more patients had a history of COPD $\left(28.6 \%\right.$ vs. $11.5 \%, \chi^{2}$ test $\left.P=.044\right)$. With regard to other relevant medical history, no statistically significant differences were identified between the two groups (Table 3).

Preoperative tumor staging with endoscopic ultrasound (uT) showed more patients with early stage disease (uTis-1, $n=11$ ) in the surgery-alone group, as opposed to none in the chemotherapy group. The number of patients with advanced (uT3-4) disease was evenly dispersed over the groups. Postoperative pathological tumor staging (pT) showed no statistically significant difference between the two groups. An equal spread of differentiation grades was observed. The number of patients with positive lymph nodes at pathological examination was slightly higher in the group that did not receive chemotherapy $(65.7 \%$ vs. $46.2 \%$ in the chemotherapy group, $\chi^{2}$ test $\left.P=.073\right)$. The majority of patients $(73.1 \%)$ in the chemotherapy group were operated on via a transthoracic approach, as opposed to approximately half of the patients $(51.4 \%)$ in the surgery-alone group $\left(\chi^{2}\right.$ test $\left.P=.039\right)$. 
TABLE 3 Baseline characteristics before treatment selection and after surgery of patients treated with surgery alone versus patients treated with surgery and perioperative chemotherapy according to the ECC regimen

\begin{tabular}{|c|c|c|c|}
\hline Characteristic & Surgery alone $(n=35)$ & Surgery and ECC $(n=52)$ & $P^{\mathrm{a}}$ \\
\hline \multicolumn{4}{|l|}{ Pretreatment data } \\
\hline Gender & & & .751 \\
\hline Male & $28(80.0 \%)$ & $43(82.7 \%)$ & \\
\hline Female & $7(20.0 \%)$ & $9(17.3 \%)$ & \\
\hline \multicolumn{4}{|l|}{ Age, years } \\
\hline Mean (SD) & $66.9(9.6)$ & $64.4(8.4)$ & $.198^{\mathrm{b}}$ \\
\hline$>60$ years & $27(77.1 \%)$ & $35(67.3 \%)$ & .320 \\
\hline $\mathrm{BMI}>27$ & $17(48.6 \%)$ & $26(50.0 \%)$ & .896 \\
\hline \multicolumn{4}{|l|}{ Medical history } \\
\hline $\mathrm{DM}$ & $7(20.0 \%)$ & $7(13.5 \%)$ & .416 \\
\hline Hypertension & $12(34.3 \%)$ & $22(42.3 \%)$ & .452 \\
\hline COPD & $10(28.6 \%)$ & $6(11.5 \%)$ & .044 \\
\hline TEE & $13(37.1 \%)$ & $12(23.1 \%)$ & .155 \\
\hline Anticoagulant therapy & $3(8.6 \%)$ & $5(9.6 \%)$ & .869 \\
\hline Smoking & $18(51.4 \%)$ & $29(55.8 \%)$ & .217 \\
\hline Ultrasound $\mathrm{T}$ stage & & & .003 \\
\hline uTis & $3(8.6 \%)$ & $0(0.0 \%)$ & \\
\hline uT1 & $8(22.9 \%)$ & $0(0.0 \%)$ & \\
\hline uT2 & $3(8.6 \%)$ & $12(23.1 \%)$ & \\
\hline uT3 & $18(51.4 \%)$ & $36(69.2 \%)$ & \\
\hline uT4 & $2(5.7 \%)$ & $2(3.8 \%)$ & \\
\hline uTx & $1(2.9 \%)$ & $2(3.8 \%)$ & \\
\hline \multicolumn{4}{|l|}{ Surgical data } \\
\hline ASA score & & & .116 \\
\hline 1 & $8(22.9 \%)$ & $13(25.0 \%)$ & \\
\hline 2 & $17(48.6 \%)$ & $34(65.4 \%)$ & \\
\hline 3 & $9(25.7 \%)$ & $5(9.6 \%)$ & \\
\hline 4 & $1(2.9 \%)$ & $0(0.0 \%)$ & \\
\hline Operative approach & & & .039 \\
\hline Transhiatal & $17(48.6 \%)$ & $14(26.9 \%)$ & \\
\hline Transthoracic & $18(51.4 \%)$ & $38(73.1 \%)$ & \\
\hline Surgery type & & & .850 \\
\hline Minimally invasive & $27(77.1 \%)$ & $41(78.8 \%)$ & \\
\hline Open & $8(22.9 \%)$ & $11(21.2 \%)$ & \\
\hline Tumor differentiation grade & & & .807 \\
\hline Poor & $14(40.0 \%)$ & $21(40.4 \%)$ & \\
\hline Moderate & $13(37.1 \%)$ & $17(32.7 \%)$ & \\
\hline Well & $1(2.9 \%)$ & $4(7.7 \%)$ & \\
\hline Unknown & $7(20.0 \%)$ & $10(19.2 \%)$ & \\
\hline Pathologic tumor stage & & & .079 \\
\hline pTis & $1(2.9 \%)$ & $0(0.0 \%)$ & \\
\hline pT0 & $0(0.0 \%)$ & $6(6.5 \%)$ & \\
\hline pT1 & $11(31.5 \%)$ & $8(15.4 \%)$ & \\
\hline pT2 & $2(5.7 \%)$ & $7(13.5 \%)$ & \\
\hline pT3 & $19(54.3 \%)$ & $30(57.7 \%)$ & \\
\hline pT4 & $2(5.7 \%)$ & $1(1.9 \%)$ & \\
\hline
\end{tabular}


TABLE 3 continued

\begin{tabular}{llll}
\hline Characteristic & Surgery alone $(n=35)$ & Surgery and ECC $(n=52)$ & $P^{\mathrm{a}}$ \\
\hline Lymph nodes & & & \\
$\quad$ Total resected, median (range) & $19(5-65)$ & $20(2-49)$ & $.396^{\mathrm{c}}$ \\
Positive lymph nodes & $23(65.7 \%)$ & $50(96.2 \%)$ & .073 \\
Radicality (R0) & $33(94.3 \%)$ & & .683 \\
Length of hospital stay, days & 17 & 15 & $.137^{\mathrm{c}}$ \\
$\quad$ Median & $10-105$ & $10-46$ \\
Range & & \\
\hline
\end{tabular}

$B M I$ body mass index, DM diabetes mellitus, ASA American Society of Anesthesiologists, $R O$ resection margin free of microscopic tumor cells

${ }^{a}$ Chi-square test unless otherwise indicated

b Two-sample $t$-test

c Mann-Whitney $U$-test

TEE

Overall, 20 patients experienced a total of 22 TEEs at some stage during the three treatment periods (Table 4). The observed TEEs included deep venous thrombosis $(n=2)$, pulmonary embolism $(n=9)$, aorta thrombosis $(n=2)$, portal vein thrombosis $(n=1)$, inferior caval vein thrombosis $(n=1)$, myocardial infarction $(\mathrm{MI} ; n=2)$, cardiac mural thrombosis $(n=1)$, and stroke (cardiovascular accident; $n=4)$.

Within the group of patients receiving chemotherapy, 17 patients $(32.7 \%)$ experienced a TEE. Three patients $(7.5 \%)$

TABLE 4 Number of TEEs during treatment in control and ECC group

\begin{tabular}{llll}
\hline Treatment period & $\begin{array}{l}\text { TEE } \\
\text { grade }\end{array}$ & $\begin{array}{l}\text { Surgery alone } \\
(n=35)\end{array}$ & $\begin{array}{l}\text { Surgery and ECC } \\
(n=52)\end{array}$ \\
\hline Preoperative & Total & $0(0.0 \%)$ & $14(29.9 \%)$ \\
& 2 & 0 & 1 \\
& 3 & 0 & 9 \\
Postoperative & 4 & 0 & 4 \\
& 5 & 0 & 0 \\
& Total & $3(8.6 \%)$ & $3(5.8 \%)$ \\
& 2 & 0 & 0 \\
& 3 & 1 & 1 \\
Postoperative & 4 & 2 & $2^{\mathrm{a}}$ \\
chemotherapy & 5 & 0 & 0 \\
& Total & NA & $2(3.8 \%)$ \\
& 2 & NA & 0 \\
& 3 & NA & 0 \\
& 4 & NA & $2^{\mathrm{b}}$ \\
& 5 & NA & 0 \\
\hline
\end{tabular}

NA not applicable

a One patient also had a preoperative TEE (grade 4)

${ }^{b}$ One patient also had a preoperative TEE (grade 3 ) were diagnosed with TEE in the surgery-alone group $\left(\chi^{2}\right.$ test $P<.01$ ). The relative risk of developing a TEE during the whole treatment period for patients treated with chemotherapy was 3.8 (95\% confidence interval $1.2-12.0 ; \chi^{2}$ test $P=.009$ ). The relative risk for of developing a TEE during the combined preoperative and postoperative period was 3.6 (95\% confidence interval 1.1-11.4; chemotherapy group, $n=16$; surgery-alone group, $n=3 ; \chi^{2}$ test $P=.014)$.

\section{Preoperative Period}

In the preoperative period, 14 patients were diagnosed with a TEE. All of these TEEs occurred in the cohort of chemotherapy patients $(26.9 \%)$.

Patients receiving chemotherapy had a follow-up CT scan for evaluation of response after the second preoperative treatment cycle. This led to coincidental detection of TEE in 6 cases. In 8 patients, the TEEs were symptomatic. The median interval between CT scan for response evaluation and surgery was 57 (range 7-91) days for patients who received chemotherapy. The median time interval between the last preoperative CT scan and surgery was 59 (range 1-92) days for the surgery-alone group (MannWhitney $U$-test $P=.915$ ).

In all cases of TEE, anticoagulant therapy was initiated. For 6 patients with preoperative TEE, chemotherapy was stopped, and in 2 cases, only cisplatin therapy was stopped (Table 5). The other 6 TEEs were discovered after the third treatment cycle. None of the patients was excluded for surgery. The median time to surgery from the last day of chemotherapy was 34 (range 13-59) days for patients who did not have a preoperative TEE and 38.5 (range 15-91) days for patients with a preoperative TEE (Mann-Whitney $U$-test $P=.239$ ).

Median postoperative hospital stay for patients with a preoperative TEE was 14 (range 11-27) days and 17 (range 
TABLE 5 Patients with preoperative TEEs and changes to their chemotherapy regimen

\begin{tabular}{lcl}
\hline Change in preoperative regimen & $n$ & Timing \\
\hline Stop chemotherapy & 1 & During first cycle \\
& 1 & During second cycle \\
& 3 & After second cycle \\
Stop cisplatin, switch to oxaliplatin & 1 & During third cycle \\
Stop cisplatin & 1 & After first cycle, completed second cycle with oxaliplatin, no third cycle \\
No change & 1 & After second cycle, completed third cycle without cisplatin \\
Total & 6 & TEE occurred after third cycle
\end{tabular}

${ }^{a}$ In 4 patients, capecitabine dose was reduced because of polyneuropathy

10-105) days for patients without TEE (Mann-Whitney $U$-test $P=.325$ ).

\section{Postoperative Period}

During the postoperative period, three TEEs occurred in the chemotherapy group (5.8\%). Another three events were recorded in the surgery-alone group $\left(8.6 \%, \chi^{2}\right.$ test $P=.613)$. None of the patients with postoperative TEE was diagnosed with atrial fibrillation. One of the patients with postoperative TEE developed a thrombus in the left ventricle during preoperative chemotherapy. In the other 5 cases, no preoperative TEEs occurred.

Median postoperative hospital stay for patients with a postoperative TEE was 23 (range 14-78) days and 15 (range 10-105) days for patients without TEE (MannWhitney $U$-test $P=.126$ ).

\section{Postoperative Chemotherapy Period}

After recovering from surgery, 21 of 52 patients continued chemotherapy. In 31 cases, postoperative chemotherapy was not administered for various reasons. Toxicity during preoperative chemotherapy formed the main reason for not receiving postoperative therapy $(n=16)$. Other reasons were metastatic disease $(n=4)$, poor patient condition $(n=6)$, death $(n=1)$, irradical resection $(n=1)$, and withdrawn consent $(n=3)$.

Two patients developed a grade 4 TEE (cardiovascular accident) during postoperative chemotherapy. One of these patients already had deep venous thrombosis and pulmonary embolism during preoperative therapy. Further postoperative chemotherapy was discontinued in both patients.

\section{Risk Factors for Thromboembolic Complications}

Besides preoperative treatment with ECC, none of the risk factors showed statistically significant correlation with TEE in univariate logistic regression analysis. To test for possible confounders, all risk factors were analyzed for correlations with the use of chemotherapy by univariate logistic regression (data not shown). Factors with a correlation $P$-value of $<.100$ were subjected to further analysis. A history of COPD and length of stay were inversely correlated with the use of preoperative chemotherapy $(P=.051$ and $P=.042$, respectively). Transthoracic surgery and the presence of positive lymph nodes in the resection specimen were positively correlated with the use of preoperative chemotherapy ( $P=.041$ and $P=.075$ respectively). To correct for possible confounding, these factors were included in multivariate logistic regression analysis.

Table 6 provides an overview of the analyzed risk factors in univariate analysis as well as the possible confounding risk factors in multivariate analysis. Preoperative chemotherapy was an independent predictor for developing TEE during the combined preoperative and postoperative period $(P=.034)$. None of the possible confounding risk factors contributed in a statistically significant way in multivariate analysis.

\section{DISCUSSION}

The current study shows that TEE is more frequent among patients selected for perioperative treatment with the ECC regimen compared to patients not receiving chemotherapy. The majority of preoperative TEEs were grade 3 and 4 , which are potentially lethal. In all cases, TEE required medical intervention with anticoagulant therapy, and in most cases the chemotherapy regimen was adjusted or discontinued. However, preoperative TEE did not disqualify patients for surgery; nor did it increase the risk of postoperative TEE.

Some studies have specifically reported on the prevalence and significance of venous thrombosis in gastroesophageal cancer patients receiving palliative chemotherapy. ${ }^{14,15}$ To date, to our knowledge, no studies have reported on the effect of TEE on perioperative outcomes in patients receiving neoadjuvant ECC therapy. This study specifically focuses on patients with EAC comparing patients who receive a specific 
TABLE 6 Univariate and multivariate analysis of the association between risk factors and development of TEE during the combined preoperative and postoperative period

\begin{tabular}{llll}
\hline Characteristic & Unadjusted OR $(95 \%$ CI), univariate & $P$ & Adjusted OR (95\% CI), multivariate $^{\mathrm{a}}$ \\
\hline Gender (male) & $0.804(0.226-2.853)$ & .735 & \\
Age (continuous) & $0.988(0.934-1.046)$ & .684 & \\
Age (>60 years) & $0.840(0.279-2.531)$ & .757 & \\
BMI (>27) & $0.900(0.325-2.492)$ & .839 & \\
TEE in history & $1.620(0.552-4.755)$ & .380 & \\
DM & $0.549(0.122-2.698)$ & .460 & \\
Hypertension & $0.659(0.224-1.944)$ & .450 & \\
COPD & $0.454(0.094-2.200)$ & .327 & $0.687(0.130-3.634)$ \\
Smoking & $1.223(0.474-3.151)$ & .677 & \\
Positive LN & $0.931(0.336-2.580)$ & .891 & $1.347(0.445-4.070)$ \\
Tumor differentiation grade & $0.958(0.682-1.345)$ & .803 & \\
LOS & $0.999(0.964-1.036)$ & .967 & $1.014(0.975-1.054)$ \\
ASA score & $0.534(0.235-1.211)$ & .133 & \\
Operation time & $1.002(0.997-1.008)$ & .458 & \\
Transthoracic surgery & $1.733(0.559-5.378)$ & .341 & $1.129(0.334-3.817)$ \\
Open surgery & $0.353(0.074-1.687)$ & .192 & .659 \\
Anticoagulant therapy & $0.484(0.056-4.199)$ & .510 & .598 \\
Chemotherapy & $4.741(1.264-17.780)$ & .021 & $4.937(1.131-21.545)$ \\
\hline CI Confice & &
\end{tabular}

$C I$ confidence interval, BMI body mass index, DM diabetes mellitus, $L N$ lymph node, $L O S$ length of hospital stay, ASA American Society of Anesthesiologists

${ }^{a}$ Included for multivariate regression analysis were possible confounders (i.e., risk factors that showed univariate association $(P<.100)$ with chemotherapy)

treatment regimen (ECC) with patients not receiving perioperative treatment in the same period. Hence, treatment standards (e.g., surgical expertise and intensive care unit protocols) were the same for all patients. However, selection bias, by which patients are selected for chemotherapy, could not be eliminated. Baseline characteristics show a less advanced endoscopically defined tumor stage (uT), but also a poorer patient condition in the surgery-alone group. Nonetheless, TEE rates are higher in the chemotherapy group. Postoperative pathologically defined tumor stage (pT), particularly for advanced disease stage (pT3), is equal in both groups. Hence, higher $\mathrm{T}$ stage does not explain the difference in TEE incidence observed in this study. Moreover, despite certain baseline differences, multivariate analysis identified chemotherapy as the only independent risk factor for preoperative and postoperative TEE.

The MAGIC trial did not describe excess thromboembolic complications during treatment. ${ }^{4}$ The authors did recommend the use of prophylactic antithrombotic therapy. The MAGIC regimen used continuous intravenous fluorouracil administration during 21 consecutive days and recommended warfarin as a prophylaxis for intravenous catheter-associated thrombosis. However, it remains unclear what percentage of patients actually received prophylaxis during the trial. Also, we may have detected more subclinical TEEs as a result of the follow-up CT scan (for response evaluation) in the chemotherapy group. Patients from the surgery-alone group did not routinely undergo a second CT scan before surgery. Nevertheless, the time interval between the last preoperative $\mathrm{CT}$ scan and surgery was equal in both groups. Furthermore, 8 out of 14 preoperative TEEs were symptomatic. The observed TEE rate in our analysis of the preoperative chemotherapy group suggests that without prophylaxis, a high TEE rate can be expected.

All patients with preoperative TEE received anticoagulant therapy until surgery. The subsequent effect on postoperative TEE cannot be measured in this study. Careful monitoring by the medical oncologist and early intervention has proved to prevent worsening of thromboembolic disease and most probably prevented postoperative events. Only 1 patient with a preoperative TEE also developed a postoperative TEE. The other 5 patients with postoperative TEE did not use anticoagulant therapy in the preoperative period.

During the last year of the inclusion period, several candidates for perioperative therapy were given an alternative regimen in which cisplatin (ECC therapy) was replaced by oxaliplatin (EOC therapy). Oxaliplatin is as effective as cisplatin in the treatment of patients with untreated advanced 
gastroesophageal cancer, but it is associated with a lower incidence of thromboembolism ${ }^{8,16,17}$

In 1 patient with preoperative TEE during the first treatment cycle, ECC was changed to EOC therapy. This patient did not receive a third treatment cycle. In another patient with TEE discovered after the second treatment cycle, cisplatin was not administered during the third cycle. In the other 6 cases, ECC therapy was changed to EOC therapy for reasons of ototoxicity $(n=2)$ and nephrotoxicity $(n=4)$. Because patients received ECC during the first treatment cycle, and on the basis of the intention-totreat principle, these patients were included in our analysis. This has possibly caused an underestimation of the TEE rate in the ECC cohort. Though speculative, it could be argued that without the alternative EOC regimen, preoperative and postoperative TEE rates would have turned out higher. It must, however, be noted that there is no evidence that oxaliplatin is preferred over cisplatin in the curative perioperative setting with respect to survival and toxicity.

Because transthoracic surgery takes more time and includes more extensive dissection than transhiatal surgery, it could be argued that operative approach might influence postoperative TEE rates. However, operative approach as a possible confounder was not correlated with TEE in multivariate analysis. With respect to postoperative recovery, no negative effects of chemotherapy were noticed. The median hospital stay was similar in both study groups. In addition, experiencing TEE during the preoperative period was not associated with increased postoperative hospital stay. Though not statistically significant, median hospital stay was increased with 7 days for patients who experienced a postoperative TEE.

Because venous thrombotic events can extend beyond the inpatient recovery period, the postoperative period included inpatient hospital stay as well as the postdischarge period up to 30 days after initial surgery. ${ }^{18}$ Despite this extended period, there was no observation of symptomatic TEE in the outpatient clinic. Moreover, there was no increased incidence of postoperative TEE within the group of patients who experienced a preoperative TEE.

Perioperative treatment with the ECC regimen improves survival in patients with EAC, but this benefit is accompanied by toxicity that could seriously harm patients and their surgical outcomes. The current study showed that TEE was frequent and was independently associated with chemotherapy. Although they were treated adequately, preoperative TEEs did not greatly influence time to surgery, hospital stay, or the occurrence of postoperative TEE. Nonetheless, in each individual case, it should be evaluated whether the benefits of the ECC regimen outweigh the increased risk of TEE. All patients who receive preoperative chemotherapy according to the
ECC regimen should undergo a follow-up CT scan after the second treatment cycle. Imaging should not only focus on treatment evaluation, but also on signs of thromboembolic disease.

OPEN ACCESS This article is distributed under the terms of the Creative Commons Attribution Noncommercial License which permits any noncommercial use, distribution, and reproduction in any medium, provided the original author(s) and source are credited.

\section{REFERENCES}

1. Cook MB, Chow WH, Devesa SS. Oesophageal cancer incidence in the United States by race, sex, and histologic type, 1977-2005. Br J Cancer. 2009;101:855-9.

2. Bosetti C, Levi F, Ferlay J, et al. Trends in oesophageal cancer incidence and mortality in Europe. Int J Cancer. 2008;122:111829

3. Lepage C, Rachet B, Jooste V, Faivre J, Coleman MP. Continuing rapid increase in esophageal adenocarcinoma in England and Wales. Am J Gastroenterol. 2008;103:2694-9

4. Cunningham D, Allum WH, Stenning SP, et al. Perioperative chemotherapy versus surgery alone for resectable gastroesophageal cancer. $N$ Engl J Med. 2006;355:11-20

5. Evans TR, Pentheroudakis G, Paul J, et al. A phase I and pharmacokinetic study of capecitabine in combination with epirubicin and cisplatin in patients with inoperable oesophago-gastric adenocarcinoma. Ann Oncol. 2002;13:1469-78

6. Rich TA, Shepard RC, Mosley ST. Four decades of continuing innovation with fluorouracil: current and future approaches to fluorouracil chemoradiation therapy. J Clin Oncol. 2004;22: 2214-32

7. Shah MA, Capanu M, Soff G, Asmis T, Kelsen DP. Risk factors for developing a new venous thromboembolism in ambulatory patients with non-hematologic malignancies and impact on survival for gastroesophageal malignancies. J Thromb Haemost. 2010;8:1702-9

8. Starling N, Rao S, Cunningham D, et al. Thromboembolism in patients with advanced gastroesophageal cancer treated with anthracycline, platinum, and fluoropyrimidine combination chemotherapy: a report from the UK National Cancer Research Institute Upper Gastrointestinal Clinical Studies Group. J Clin Oncol. 2009;27:3786-93

9. Agnelli G, Bolis G, Capussotti L, et al. A clinical outcome-based prospective study on venous thromboembolism after cancer surgery: the @ RISTOS project. Ann Surg. 2006;243:89-95

10. Khorana AA, Connolly GC. Assessing risk of venous thromboembolism in the patient with cancer. J Clin Oncol. 2009;27: 4839-47

11. Oken MM, Creech RH, Tormey DC, et al. Toxicity and response criteria of the Eastern Cooperative Oncology Group. Am J Clin Oncol. 1982;5:649-55

12. Schuurmans H, Steverink N, Lindenberg S, Frieswijk N, Slaets JP. Old or frail: what tells us more? J Gerontol A Biol Sci Med Sci. 2004;59:M962-5

13. National Cancer Institute common terminology criteria for adverse events v.4.03. U.S. Department of Health and Human Services. http://evs.nci.nih.gov/ftp1/CTCAE/About.html

14. Tesselaar ME, Ouwerkerk J, Nooy MA, Rosendaal FR, Osanto S. Risk factors for catheter-related thrombosis in cancer patients. Eur J Cancer. 2004;40:2253-9

15. Tetzlaff ED, Correa AM, Baker J, Ensor J, Ajani JA. The impact on survival of thromboembolic phenomena occurring before and 
during protocol chemotherapy in patients with advanced gastroesophageal adenocarcinoma. Cancer. 2007;109:1989-95

16. Cunningham D, Starling N, Rao S, et al. Capecitabine and oxaliplatin for advanced esophagogastric cancer. $N$ Engl J Med. 2008;358:36-46

17. Montagnani F, Turrisi G, Marinozzi C, Aliberti C, Fiorentini G. Effectiveness and safety of oxaliplatin compared to cisplatin for advanced, unresectable gastric cancer: a systematic review and meta-analysis. Gastric Cancer. 2011;14:50-5

18. Fleming FJ, Kim MJ, Salloum RM, Young KC, Monson JR. How much do we need to worry about venous thromboembolism after hospital discharge? A study of colorectal surgery patients using the National Surgical Quality Improvement Program database. Dis Colon Rectum. 2010;53:1355-60 